Elżbieta Kotlicka, Institute of Mathematics, Łódź Technical University, al. Politechniki 11, I-2, 90-924 Łódź, Poland. e-mail: ekot@ck-sg.p.lodz.pl

\title{
THE DOUBLE DIFFERENCE PROPERTY FOR SOME CLASSES OF FUNCTIONS
}

\author{
Abstract \\ Using some results of M. Laczkovich and T. Keleti we show that for \\ several classes of functions the double difference property holds.
}

The notions of the difference property and the double difference property for various classes of real functions were investigated by several authors. (See e.g. [1], [5], and [6].) Some new ideas connected with the difference property are contained in the recent results of Keleti ([3], [4]). We develop those studies and obtain new theorems showing that certain classes of functions have the double difference property.

We will consider the additive group $\mathbb{G}$ equal to $\mathbb{R}$ or $\mathbb{T}$ where $\mathbb{T}$ is the circle group $\mathbb{R} / \mathbb{Z}$ (and $\mathbb{Z}$ stands for the set of all integers). Functions defined on $\mathbb{T}$ can be treated as functions defined on $\mathbb{R}$ and being periodic with period 1 . We will study pairs $\left(\mathcal{F}, \mathcal{F}^{(2)}\right)$ where $\mathcal{F}$ and $\mathcal{F}^{(2)}$ will always be classes of functions on $\mathbb{G}$ and $\mathbb{G}^{2}$, respectively. Usually $\mathcal{F}$ and $\mathcal{F}^{(2)}$ will be classes of one and two variable functions having a given property. For a fixed function $f: \mathbb{G} \rightarrow \mathbb{R}$ and any $h \in \mathbb{G}$ we define the difference function $\Delta_{h} f: \mathbb{G} \rightarrow \mathbb{R}$ by

$$
\Delta_{h} f(x)=f(x+h)-f(x),
$$

and the double difference function $D f: \mathbb{G}^{2} \rightarrow \mathbb{R}$ by

$$
D f(x, y)=f(x+y)-f(x)-f(y) .
$$

Recall that a class $\mathcal{F}$ (respectively, a pair of classes $\left(\mathcal{F}, \mathcal{F}^{(2)}\right)$ ) is said to have the difference property (respectively, the double difference property), if every function $f: \mathbb{G} \rightarrow \mathbb{R}$ such that $\Delta_{h} f \in \mathcal{F}$ (respectively, $D f \in \mathcal{F}^{(2)}$ ) for each $h \in \mathbb{G}$, is of the form $f=g+H$ where $g \in \mathcal{F}$ and $H$ is an additive

Key Words: the difference functions, the difference property, the double difference property

Mathematical Reviews subject classification: 26A99, 39A70, 26B35

Received by the editors December 18, 1998 
function, that is, $H(x+y)=H(x)+H(y)$ for every $x, y \in \mathbb{R}$. Our Proposition 1 shows some connection between these two properties.

A class $\mathcal{F}$ is called translation invariant if, for any $f \in \mathcal{F}$ and $a, b \in \mathbb{G}$, the function $g(x):=f(x+a)+b, x \in \mathbb{G}$, belongs to $\mathcal{F}$.

Let $\mathcal{I}$ be a translation invariant, proper $\sigma$-ideal on $\mathbb{G}$. We are most interested in the special cases of null and the first category sets. Denote these ideals by $\mathcal{N}$ and $\mathcal{M}$. We say that a property $P(x)$ holds $\mathcal{I}$-almost everywhere or for $\mathcal{I}$-almost all $x$, if it holds for all points $x \in \mathbb{G}$ except for some of them which form a set in $\mathcal{I}$. A pair $\left(\mathcal{F}, \mathcal{F}^{(2)}\right)$ is called $\mathcal{I}$-hereditary if $\mathcal{I}$-almost all sections $f^{y}$ are in $\mathcal{F}$ for every $f \in \mathcal{F}^{(2)}$ (where $f^{y}(x)=f(x, y), x \in \mathbb{G}$ ). We say that a pair $\left(\mathcal{F}, \mathcal{F}^{(2)}\right)$ is hereditary if there exists an $\mathcal{I}$ such that $\left(\mathcal{F}, \mathcal{F}^{(2)}\right)$ is $\mathcal{I}$-hereditary.

Lemma 1. Suppose that $\mathcal{F}$ is a translation invariant additive group of functions $f: \mathbb{G} \rightarrow \mathbb{R}$ and $\left(\mathcal{F}, \mathcal{F}^{(2)}\right)$ is hereditary. Then the condition $D f \in \mathcal{F}^{(2)}$ implies that all difference functions $\Delta_{h} f$ are in $\mathcal{F}$.

Proof. (Cf. [5] Theorem 5.) Suppose that $D f \in \mathcal{F}^{(2)}$. By the assumption of hereditarity of $\left(\mathcal{F}, \mathcal{F}^{(2)}\right)$ there exists a translation invariant, proper $\sigma$-ideal $\mathcal{I}$ and a set $Y \in \mathcal{I}$, such that $(D f)^{y} \in \mathcal{F}$ for any $y \in Y^{c}$ (where $Y^{c}$ stands for the complement of $Y)$. Since $Y^{c} \cap\left[\left(-Y^{c}\right)+h\right] \neq \emptyset$ for any $h \in \mathbb{G}$, there are $y_{1}, y_{2} \in Y$ such that $h=y_{1}+y_{2}$. Thus

$\Delta_{h} f(x)=f(x+h)-f(x)=f\left(x+y_{1}+y_{2}\right)-f(x)$

$=\left(f\left(x+y_{1}+y_{2}\right)-f\left(x+y_{2}\right)-f\left(y_{1}\right)\right)+\left(f\left(x+y_{2}\right)-f\left(y_{2}\right)-f(x)\right)+f\left(y_{1}\right)+f\left(y_{2}\right)$

$=(D f)^{y_{1}}\left(x+y_{2}\right)+(D f)^{y_{2}}(x)+f\left(y_{1}\right)+f\left(y_{2}\right)$,

so $\Delta_{h} f \in \mathcal{F}$ for each $h \in \mathbb{G}$.

The following proposition is an immediate consequence of Lemma 1.

Proposition 1. Assume that $\mathcal{F}$ is a translation invariant additive group of functions $f: \mathbb{G} \rightarrow \mathbb{R}$ and $\left(\mathcal{F}, \mathcal{F}^{(2)}\right)$ is hereditary. If $\mathcal{F}$ has the difference property then the pair $\left(\mathcal{F}, \mathcal{F}^{(2)}\right)$ has the double difference property.

Remark. Notice that the inverse implication is false. For example, the family $\mathcal{B}$ of all bounded functions satisfies the assumptions of Proposition 1, the pair $\left(\mathcal{B}, \mathcal{B}^{(2)}\right)$ has the double difference property, but $\mathcal{B}$ does not have the difference property [1].

We consider the following classes of real-valued functions on $\mathbb{G}$ :

- $\mathcal{C}$ - the continuous functions,

- $\mathcal{A} p \mathcal{C}$ - the approximately continuous functions (see [8], pp. 131-132),

- $\mathcal{P D}$ - the pointwise discontinuous functions (i.e., those having a dense set of continuity points). 
Theorem 1. The pairs $\left(\mathcal{C}, \mathcal{C}^{(2)}\right),\left(\mathcal{A} p \mathcal{C}, \mathcal{A p C}^{(2)}\right)$ and $\left(\mathcal{P D}, \mathcal{P} \mathcal{D}^{(2)}\right)$ have the double difference property.

Proof. All considered classes of functions (of one variable) are translation invariant additive groups and have the difference property (see respectively, [1], [5] and [6]), so it is enough to check that the assumption about hereditarity is fulfilled. In the case of $\left(\mathcal{C}, \mathcal{C}^{(2)}\right)$ this is obvious and in the case of $\left(\mathcal{A p C}, \mathcal{A p C}^{(2)}\right)$ it directly follows from Theorem 4 in [2] (we consider $\mathcal{I}=\{\emptyset\}$ ). We will show that the pair $\left(\mathcal{P D}, \mathcal{P} \mathcal{D}^{(2)}\right)$ is $\mathcal{M}$-hereditary. Let $f \in \mathcal{P D}^{(2)}$. Denoting by $C_{f}$ the set of continuity points of $f$, we have that $C_{f}$ is a dense $G_{\delta}$ subset of $\mathbb{R}^{2}$. By the Kuratowski-Ulam theorem [7], for $\mathcal{M}$-almost all $y \in \mathbb{R}$, the sections $\left(C_{f}\right)^{y} \subset C_{\left(f^{y}\right)}$ are dense in $\mathbb{R}$, which means that $\mathcal{M}$-almost all sections $f^{y}$ are in $\mathcal{P D}$.

Let $\mathcal{F}$ and $\mathcal{G}$ be classes of real-valued functions on $\mathbb{G}$ with $\mathcal{G} \subset \mathcal{F}$. Tamas Keleti in his doctoral dissertation [3] considered the family $\mathcal{H}(\mathcal{F}, \mathcal{G})=\{A \subset$ $\left.\mathbb{G}: \exists f \in \mathcal{F} \backslash \mathcal{G} \quad \forall h \in A \quad \Delta_{h} f \in \mathcal{G}\right\}$ consisting of sets $A$ which do not satisfy the condition:

$$
(*) \quad \forall f \in \mathcal{F}\left(\left(\forall h \in A \quad \Delta_{h} f \in \mathcal{G}\right) \Rightarrow f \in \mathcal{G}\right) .
$$

Keleti established $\mathcal{H}(\mathcal{F}, \mathcal{G})$ for several classes of measurable functions and gave a characterization of the difference property in the language of property $(*)$ ([4], Lemma 1.1). In a similar way we shall characterize the double difference property, replacing $(*)$ by the following condition:

$$
(* *) \quad \forall f \in \mathcal{F}\left(D f \in \mathcal{G}^{(2)} \Rightarrow f \in \mathcal{G}\right) .
$$

Theorem 2. Let $\mathcal{F}$ be an additive group of functions, let $\mathcal{G}$ be a subgroup of $\mathcal{F}$ containing all linear functions and $\mathcal{G}^{(2)} \subset \mathcal{F}^{(2)}$. Assume that

(i) $\left(\mathcal{F}, \mathcal{F}^{(2)}\right)$ has the double difference property,

(ii) every additive function from $\mathcal{F}$ is linear.

Then the following conditions are equivalent:

(a) $(\mathcal{F}, \mathcal{G})$ satisfies condition $(* *)$,

(b) $\left(\mathcal{G}, \mathcal{G}^{(2)}\right)$ has the double difference property.

Proof. (a) $\Rightarrow$ (b): Let $f$ be a function from $\mathbb{G}$ to $\mathbb{R}$ such that $D f \in \mathcal{G}^{(2)}$. Since $\mathcal{G}^{(2)} \subset \mathcal{F}^{(2)}$ and the pair $\left(\mathcal{F}, \mathcal{F}^{(2)}\right)$ has the double difference property, there exist a $g \in \mathcal{F}$ and an additive function $H$ such that $f=g+H$. Thus $D g=D f$ belongs to $\mathcal{G}^{(2)}$, so by assumption (a) we have that $g \in \mathcal{G}$. 
(b) $\Rightarrow\left(\right.$ a): Let $f \in \mathcal{F}$ and $D f \in \mathcal{G}^{(2)}$. The double difference property of the pair $\left(\mathcal{G}, \mathcal{G}^{(2)}\right)$ implies that $f=g+H$ where $g \in \mathcal{G}=$ and $H$ is additive. Since $H=f-g$ belongs to $\mathcal{F}$, so by (ii) $H$ is a linear function. Thus $f$, as a sum of two functions from $\mathcal{G}$, has to be in $\mathcal{G}$.

Note that under assumptions of Lemma 1 the condition $\mathbb{G} \notin \mathcal{H}(\mathcal{F}, \mathcal{G})$ implies that the pair $(\mathcal{F}, \mathcal{G})$ fulfills condition $(* *)$. Omitting assumptions which were not used in the proof of the implication " $(\mathrm{a}) \Rightarrow(\mathrm{b})$ " we have:

Corollary 1. Assume that $\mathcal{G} \subset \mathcal{F}$ is a translation invariant additive group of functions, $\mathcal{G}^{(2)} \subset \mathcal{F}^{(2)}$ and $\left(\mathcal{G}, \mathcal{G}^{(2)}\right)$ is hereditary. If $\mathbb{G} \notin \mathcal{H}(\mathcal{F}, \mathcal{G})$ and the pair $\left(\mathcal{F}, \mathcal{F}^{(2)}\right)$ has the double difference property then $\left(\mathcal{G}, \mathcal{G}^{(2)}\right)$ has this property, too.

In the sequel by $L_{0}$ we denote the class of all Lebesgue measurable functions on $\mathbb{G}$ and by $M_{0}$ - the class of functions with the Baire property on $\mathbb{G}$. For a fixed family $\mathcal{F}$ we define

$$
\mathcal{F}_{\mathcal{I}}=\left\{f \in \mathbb{R}^{\mathbb{G}}: \exists g \in \mathcal{F} \quad f=g \quad \mathcal{I} \text { - almost everywhere }\right\},
$$

and we denote by $\mathcal{C}_{\mathcal{I}}$ the family of $\mathcal{I}$-essentially continuous functions and by $\mathcal{B}_{\mathcal{I}}$ the family of $\mathcal{I}$-essentially bounded functions. Let also $L_{\infty}=L_{0} \cap \mathcal{B}_{\mathcal{N}}$.

Theorem 3. The pairs $\left(\mathcal{C}_{\mathcal{N}}, \mathcal{C}_{\mathcal{N}}^{(2)}\right)$ and $\left(L_{p}(\mathbb{T}), L_{p}^{(2)}(\mathbb{T})\right)($ for $0<p \leq \infty)$ have the double difference property.

Proof. It suffices to apply Corollary 1 in the case when $\mathcal{F}=L_{0}$ and $\mathcal{G}=$ $\mathcal{C}_{\mathcal{N}}$ (or respectively, $\mathcal{G}=L_{p}(\mathbb{T})$ ). The Fubini theorem implies that respective pairs are $\mathcal{N}$-hereditary. Using the known facts:

- the pair $\left(L_{0}, L_{0}^{(2)}\right)$ has the double difference property (proved by Laczkovich in [5]),

- $\mathbb{R} \notin \mathcal{H}\left(L_{0}, \mathcal{C}_{\mathcal{N}}\right)$ and $\mathbb{T} \notin \mathcal{H}\left(L_{0}, L_{p}\right)$ for $0<p \leq \infty$ (results of Keleti [4]),

we have the assertion.

Remark. One can consider the category analogs of the pairs $\left(\mathcal{C}_{\mathcal{N}}, \mathcal{C}_{\mathcal{N}}^{(2)}\right)$ and $\left(L_{\infty}(\mathbb{T}), L_{\infty}^{(2)}(\mathbb{T})\right)$ and can ask whether they have the double difference property. That would be true if the following problem had the positive answer: Problem. Does the pair $\left(M_{0}, M_{0}^{(2)}\right)$ have the double difference property? Acknowledgements. The author would like to thank the referee for several valuable comments. 


\section{References}

[1] N.G. de Bruijn, Functions whose differences belong to a given class, Nieuw Arch. Wisk. 23(1951), 194-218.

[2] C. Goffman, C. J. Neugebauer, T. Nishiura, Density topology and approximate continuity, Duke Math. J. 28(1961), 497-506.

[3] T. Keleti, Difference functions of periodic measurable functions, $\mathrm{PhD}$ thesis, Eötvös Loránd University, Budapest, 1996 (http://www.cs.elte.hu/phd_th/).

[4] T. Keleti, Difference functions of periodic measurable functions, Fund. Math. 157(1998), 15-32.

[5] M. Laczkovich, Functions with measurable differences, Acta Math. Hungar. 35(1980), 217-235.

[6] M. Laczkovich, On the difference property of the class of pointwise discontinuous functions and some related classes, Canad. J. Math. 36(1984), 756-768.

[7] J.C. Oxtoby, Measure and Category, Springer, New York, 1971.

[8] S. Saks, Theory of the Integral, G. E. Stechert, New York, 1937. 
